\title{
TRAIL Immunolocalisation in the Rat Periodontal Ligament during Experimental Tooth Movement. A Preliminary Study
}

\author{
Carla Loreto $^{1}$, Giuseppe Musumeci ${ }^{1}$, Sergio Castorina ${ }^{1}$, Jessica Valentino ${ }^{2}$, Salvatore Giunta ${ }^{1}$, \\ Rosalia Leonardi ${ }^{2}$ \\ ${ }^{1}$ Department of Bio-Medical Sciences, Anatomy and Histology Section, University of Catania, Catania, Italy \\ ${ }^{2}$ Department of Orthodontics, University of Catania, Catania, Italy \\ Email: "g.musumeci@unict.it
}

Received March 26, 2013; revised May 22, 2013; accepted June 4, 2013

Copyright $(2013$ Carla Loreto et al. This is an open access article distributed under the Creative Commons Attribution License, which permits unrestricted use, distribution, and reproduction in any medium, provided the original work is properly cited.

\begin{abstract}
The aim of this study was to evaluate immunohistochemically TRAIL (TNF-Related Apoptosis Inducing Ligand) expression in the periodontal ligament of rats' molars considering its distribution pattern in the tension and compressive regions of ligament during orthodontic movement. Sixteen Sprague-Dawley rats, weighing between 120 and $200 \mathrm{~g}$ were used in the present study. Tooth movement was induced by placing elastic bands between the maxillary first and second molars. The elastic bands were left in place for 12 (time T1) and 24 (time T2) hours. After rats' euthanasia, maxillae were dissected free and the sections were exposed to a primary rabbit polyclonal anti-TRAIL anti-body. The staining status was identified as either negative or positive by using 3,3'-diaminobenzidine (DAB) to develop the immunoreaction. Data were analyzed using ANOVA test and a post-hoc Dunn's Multiple Comparison test. P-values of less than 0.05 were considered statistically significant. The staining for TRAIL in the tension side appeared darker (intensity of staining = 2) than in the compressive side (intensity of staining =1) $12 \mathrm{~h}$ after the tooth movement. The stainings of both sides of PDL were observed to be up-regulated $24 \mathrm{~h}$ after the application of the force (tension side $=3$, compression side $=2 ; \mathrm{P}<0.05$ ). TRAIL is expressed in the periodontal ligament of rats' molars during experimental tooth movement both in the compression and in the tension sides. The latter showed the highest amount of TRAIL immunolabeling.
\end{abstract}

Keywords: Immunohistochemistry; Pperiodontal Ligament; Tooth Movement; Trail

\section{Introduction}

Orthodontic tooth movement (OTM) is characterized by remodelling changes in dental pulp, periodontal ligament (PDL), alveolar bone and gingiva in response to a mechanical loading [1,2]. The use of an external force in order to induce tooth movement determines cellular responses that produce selective adaptation of the alveolus to support the migration of teeth. During this process, periodontal tissues could be divided into two opposing distinct sides named tension side (where the displacement of the dental root creates a strain force in the PDL fibers) and compression side (where a compression force is created by root against the bone and the fibers are unloaded) $[2,3]$. The assumption that the tensile and compressive forces predominate in opposing sides of PDL

*Corresponding author. during orthodontic movement and trigger different patterns of tissue remodeling is reasonable. This latter is associated with the differential expression of bone remodeling markers [4-6] and with the pattern of tooth displacement observed in vivo [7]. Osteoclast and osteoblast are the key-cells during these processes. In fact, their differential activities are required to allow proper coupling of bone resorption and deposition in compression and tension regions in the PDL, respectively [8]. The appearance of osteoclasts is the indispensable first step in orthodontic tooth movement because they are the only cells responsible for bone resorption. Osteoclasts activity and the consequent bone turnover are mediated by the production of pro-osteoclastic inflammatory cytokines such as ligand of receptor activator of nuclear factor $\kappa$ (RANKL), interleukin (IL)-1 $\beta$, prostaglandin $(\mathrm{PG}) \mathrm{E}_{2}$, IL-6 and tumor necrosis factor (TNF)- $\alpha$ [9]. Another 
molecule that performs a key-role in osteoclast life cycle is TRAIL (TNF-Related Apoptosis Inducing Ligand). Even if the function of RANKL and the others pro-osteoclastic inflammatory cytokines have been widely studied [10-12], literature is lack of evidence about TRAIL importance during OTM. This molecule is a component of the TNF ligand super family, which exhibits potent cytotoxic activity and induce apoptosis of susceptible cells $[13,14]$ but some evidences show that TRAIL could have a regulatory role in various tissues such as neurons, hepatocytes and osteoclasts and other tissues [15-18]. Accordingly the aim of this study was to evaluate immunohistochemically TRAIL expression in the PDL of rat molars considering its distribution pattern in the tension and compressive regions of PDL during orthodontic movement.

\section{Materials and Methods}

\subsection{Animals}

Sixteen Sprague-Dawley rats, weighing between 120 and $200 \mathrm{~g}$ were used in the present study. The experimental side was randomly chosen and was subjected to therapy while the contralateral side served as a control. The experimental procedures have been described in detail previously [5]. Briefly, tooth movement was induced by placing elastic bands between the maxillary first and second molars. This action created compression and tension areas around the roots of the first and second molars and produced a force of about 5N [19]. The elastic bands were left in place for 12 (time T1) and 24 (time T2) hours. Rats were killed by intracardial perfusion with a fixative solution of $4 \%$ paraformaldehyde, $\mathrm{pH}$ 7.2. After perfusion, the maxillae were dissected free. All procedures were performed under anesthesia using a mixture of ketamine $(90 \mathrm{mg} / \mathrm{kg}$, Abbott Laboratories, North Chicago, IL) and Xylazine (5 mg/kg, Burns Veterinary Supply, Rockville, MD). Experiment was performed in accordance with the European Communitie Council Directive (86/609/EEC) and Italian Animal Protection Law (116/1992).

\subsection{Immunohistochemistry (IHC)}

Molar-bearing segments of alveolar bone were cut from each side and further fixed in separate jars containing 50 $\mathrm{ml}$ of $4 \%$ paraformaldehyde overnight at $41^{\circ} \mathrm{C}$. The specimens were decalcified in $10 \%$ ethylenediaminetetraacetic acid (EDTA, Sigma, St. Louis, MO), pH 7.2, for 6 - 8 weeks. The decalcified tissue specimens were dehydrated through ascending concentrations of ethanol. Subsequently, the specimens were labelled and processed for embedding in paraffin wax by routine protocol. Oblique, serial, 4-6-mm-thick sections, including the crestal areas mesial and distal to the maxillary molars, were cut by microtome. The sections were mounted on L-polylysine-coated glass slides (Corning, Acton, MA). Tooth tissue sections were dewaxed by immersion in two changes of xylene and then rehydrated through a graded ethanol series to double-distilled water. Tissues were processed as previously described [20]. Briefly, to inhibit endogenous peroxidase activity, they were incubated with $3 \% \mathrm{H}_{2} \mathrm{O}_{2}$ in methanol at $4^{\circ} \mathrm{C}$ for $15 \mathrm{~min}$, then rinsed for $20 \mathrm{~min}$ with phosphate-buffered saline (PBS; BioOptica, Milan, Italy). Specimens were then treated with $5 \%$ bovine serum albumin (BSA) in PBS for 1 hour to prevent non-specific binding of the antibody. After blocking, slides were incubated overnight with a primary rabbit polyclonal anti-TRAIL anti-body (Santa Cruz Biotechnology, Inc., Santa Cruz, CA, USA) used at 1:200 working dilutions. Detections was performed by using 3,3'-diaminobenzidine (DAB, Dako Glostrup, Denmark) as chromogen (LSAB 2 System-HRB, Dako, Glostrup, Denmark). Sections were stained for alkaline phosphatase (AP) as a marker for active osteoblasts or tartrateresistant acid phosphatase (TRAP) for differentiated osteoclasts and osteoclast precursors. Sections were counter-stained with hematoxylin. Subsequent stages were performed at room temperature. Slides were then washed and mounted in PBS/glycerol (50:50) and placed on glass microscope slides. The sections were observed by using a light microscope (Zeiss Axioplan, Berlin, Germany) and photographed with a digital camera (Canon, Hong Kong, Japan).

\subsection{Evaluation of Immunohistochemistry}

The TRAIL staining status was identified as either negative or positive by using 3,3'-diaminobenzidine (DAB) to develop the immunoreaction. Intensity of staining (IS) was graded on a scale of $0-4$, according to the following assessment: $0=$ no detectable staining, $1=$ weak staining, 2 = moderate staining, $3=$ strong staining, $4=$ very strong staining. The percentage of TRAIL expression $($ Extent Score $=$ ES) was independently evaluated by 3 investigators (2 anatomical morphologists and one histologist) and scored as a percentage of the final number of 100 in five categories: $0=<5 \%$; $+=5 \%-30 \%$; $++=$ $31 \%-50 \%$; $+++=51 \%-75 \%$, and $++++=>75 \%$. Counting was performed at X200 magnification.

\subsection{Positive and Negative Controls}

Positive and negative controls were performed to test the specific reaction of primary antibodies at the protein level. For positive control testing basal cell carcinoma tissue was exposed to an immunoperoxidase process. Immunolabeling for TRAIL was found in both membranes and cytoplasm. For negative controls, slides were treated with normal rabbit serum instead of the specific anti- 
bodies.

\subsection{Statistical Analysis}

Mean values and standard deviations were obtained for the PDL portions examined. Data were further analyzed using ANOVA test and a post-hoc Dunn's Multiple Comparison test. P-values of less than 0.05 were considered statistically significant. All data were analyzed with the SPSS program (SPSS ${ }^{\circledR}$ release 16.0, Chicago, IL, USA). Cohen's $\mathrm{k}$ was applied to measure the agreement between the three observers and averaged over all three to evaluate overall agreement using the following grading: 0 - 0.2 (slight), $0.21-0.40$ (fair), 0.41 - 0.60 (moderate), $0.61-0.80$ (substantial), and $0.81-1.0$ (almost perfect).

\section{Results}

Histological examination revealed that tooth movement was associated with compression and tension areas. In the experimental sections, the PDL fibers were disoriented on the strain and compressive sides. Osteoclasts appeared both in the compression and tension sides. Specimens of PDL of rats' molar showed a significant increase of TRAIL expression with the immunohistochemical analysis (Figure 1) at T1 and T2 respect to the control $(\mathrm{P}<0.05)$. The highest levels of TRAIL expression were revealed in the tension side. The staining for TRAIL in the tension side appeared darker than in the compressive side $12 \mathrm{~h}$ after the tooth movement. The stainings of both sides of PDL were observed to be upregulated $24 \mathrm{~h}$ after the application of the force. The PDL cells showed a time and side dependent immunolabeling pattern for TRAIL. At time T1 (Figure 2), this molecule was moderately expressed in the tension side (IS: 2; ES: $++)$ and faintly present on the compression side (IS: 1 ; ES: ++). At time T2 (Figure 3), TRAIL was clearly detected both on the compression side and tension side, although with different scores. In fact in the first one, extent score showed a weak reactivity (ES: +++$)$ while in the second one showed a strong reactivity (ES: +++). Concerning IS values at $\mathrm{T} 2$, the compression side had a score of 3 and tension side had a score of 2. Interobserver agreement measured as $\mathrm{k}$ coefficient was 0.90 (almost perfect). Figures 2 and 3 illustrate the immunostaining reaction in the experimental (tension and compressive sides) group.

\section{Discussion}

Osteoclasts recruitment and activation are part of a complex process accomplished through an orderly sequence of events: 1) stem cell division and osteoclast progenitor proliferation in hematopoietic tissues, 2) migration of mononuclear osteoclast precursors to sites of bone resorp-

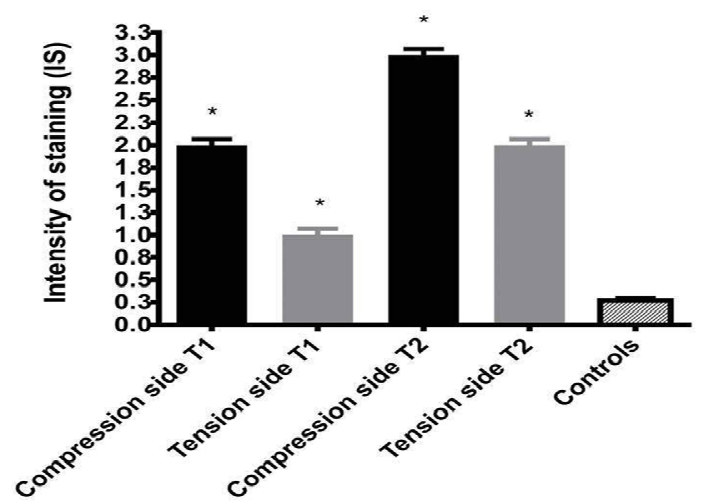

Figure 1. TRAIL intensity of staining on compression side, tension side and control group at T1 $(12 \mathrm{~h})$ and T2 (24 h). $*=\mathbf{P}<\mathbf{0 . 0 5}$.

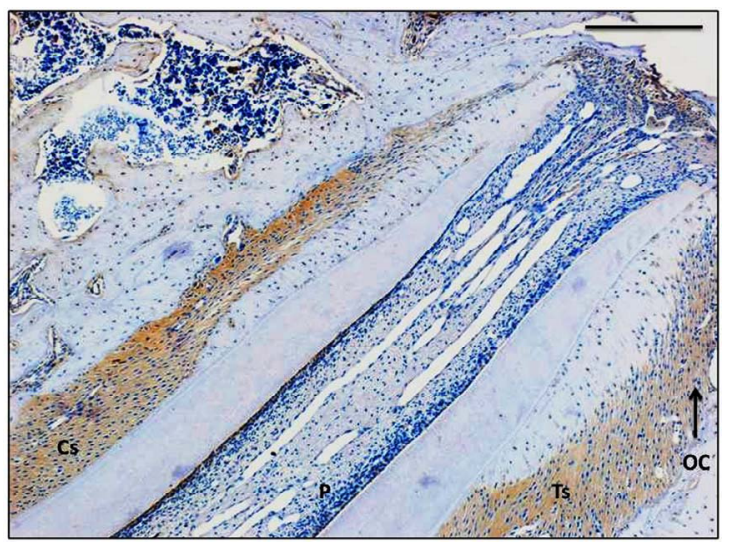

Figure 2. TRAIL immunolabelling at T1: sagittal section of section of maxillary first molars. TRAIL was moderately expressed in the tension side (IS: 2 ; ES: ++ ) and faintly detectable on the compression side (IS: 1 ; ES: ++ ). Bar $=200$ $\mu \mathrm{m}$. OC: osteoclast; Ts: tension side; Cs: compression side; P: pulp.

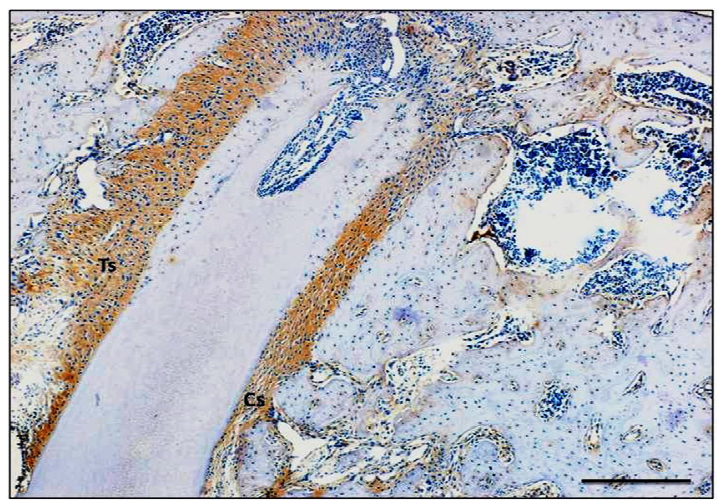

Figure 3. TRAIL immunolabeling at T2: sagittal section of section of maxillary first molars. TRAIL was clearly detected both on the compression side and tension side, although with different scores. In fact in compression side the IS score was 2 with ES: +++, while in the tension side a score of 3 and ES: +++ was observed. Bar $=200 \mu \mathrm{m}$. Ts: tension side; Cs: compression side. 
tion, 3) differentiation into committed osteoclast precursors (pre-osteoclasts) guided by cell-to-cell interaction with osteoblasts, and 4) fusion into multinuclear osteoclasts [8]. Even if many studies confirmed osteoclasts originate from cells away from PDL [8,21], several authors demonstrated that during OTM the number of these large multinucleated cells increases in the PDL near the compression side $[3,21,22]$. Several molecules play a role into their life cycle and it is supposed that TRAIL could have an important function. Accordingly, the aim of the present study was to evaluate the expression of TRAIL in rat PDL in order to detect the possible relationship between osteoclast activation and turnover and TRAIL induction. TRAIL is a type II trans-membrane protein that induces apoptosis by binding to its death domain-containing receptors, DR4 and DR5 [13,15,23-30]. Furthermore, TRAIL interacts with at least three "decoy" receptors, without the death effector domains, which are also known as DcR1, DcR2 and osteoprotegerin. In TRAIL-sensitive cells, their expression protects cells from TRAIL-induced apoptosis [13,15,31,32]. We conduced our research on two groups (experimental and control groups) at two different times: after 12 hours (time T1) and after one day of treatment (time T2). The results of our analysis confirmed a higher expression of TRAIL in the experimental sample when compared with specimens in the control group. This outcome was statistically significant both at $\mathrm{T} 1$ and $\mathrm{T} 2(\mathrm{P}<0.05)$. A detailed analysis of the expression of TRAIL revealed an interesting evidence: TRAIL was found both in the tension and in the compression side of PDL, even if higher levels of immunolabeling were reported in the tension regions. This important finding could suggest that TRAIL plays a dual role during OTM, probably depending on which receptor it is able to bind. In fact, in the compression side, where the osteoclastic activity is required in order to allow bone resorption, it may be hypothesized that TRAIL could join the decoy receptors DcR1 and DcR2 in order to induce osteoclastic differenttiation and activation. This result supports Kumamoto statements in ameloblastomas [16]. In the tension side, where the alveolar bone deposition prevails, TRAIL increased expression could be related to the activation of death receptors DR4 and DR5. This expression could promote the apoptosis chain that leads to osteoclasts programmed cell death. The importance of this evidences consist that would be reasonable to speculate that orthodontic movement may proceed without stopping as long as active osteoclastic population remains actively within the periodontal space. The limit to this process should be represented by osteoclast death: the understanding of the biological events that underlie OTM may show a crucial importance in clinical practice. In a futuristic perspective, the knowledge of the substances that set in dental move- ment and the detection of their site of action, would allow to determine, accurately, the beginning and the conclusion of the active therapy. Clinicians could control these phases by administering molecules capable of inducing cell death in order to stop tooth movement or promoting cell differentiation to accelerate OTM as suggested by Sekhavat [33], Yamasaki [34], and Leiker [35]. Hypothesizing that TRAIL has a dual role during tooth movement and thus may be able to selectively activate or inhibit bone resorption, its administration in vivo could be helpful in order to obtain a better control of OTM. More scientific evidence in this field could be useful to a better understanding of the balance between resorption and deposition, which is the equilibrium that characterizes bone tissue and plays an essential role in orthodontic movement [36]. Studies on the osteoclasts life cycle may suggest interesting implications for the assessment of the timing for orthodontic treatment (i.e. discovering the behaviour of OTM cells, clinicians could program plans of activation and reactivation of the appliances). Improving our knowledge of these biological processes, includeing cell recruitment and clearance, the efficiency of orthodontically treated tooth movement could reach higher levels. In conclusion TRAIL is expressed in the PDL of rat molars during experimental tooth movement both in the compression and in the tension sides. Tension side showed the highest amount of TRAIL immunolabeling. Further studies should be conducted in order to assess TRAIL influence on other kind cells during OTM and to study the co-localization of death and decoy TRAIL receptors on tension and compressive side, in order to acquire a global view of this process and to provide candidates for future study on this topic.

\section{Acknowledgements}

The study was funded by Department of Bio-Medical Sciences, University of Catania.

\section{REFERENCES}

[1] S. A. Alfaqeeh and S. Anil, "Osteocalcin and N-Telopeptides of Type I Collagen Marker Levels in Gingival Crevicular Fluid during Different Stages of Orthodontic Tooth Movement, ” Americam Journal Orthodontics and Dentofacial Orthopedics, Vol. 139, No. 6, 2011, pp. 553559. doi:10.1016/j.ajodo.2011.03.005

[2] T. P. Garlet, U. Coelho, C. E. Repeke, J. S. Silva, Q. Cunha Fde and G. P. Garlet, "Differential Expression of Osteoblast and Osteoclast Chemmoatractants in Compression and Tension Sides during Orthodontic Movement, ” Cytokine, Vol. 42, No. 3, 2008, pp. 330-335. doi:10.1016/j.cyto.2008.03.003

[3] R. B. Johnson, “Osteoclast Distribution within the Rat Interdental Septum Coincident to Experimental Tooth Movement Using Light Forces,” The Anatomical Records, 
Vol. 290, No. 1, 2007, pp. 74-82. doi:10.1002/ar.20408

[4] R. Leonardi, C. Loreto, N. F. Talic, R. Caltabiano and G. Musumeci, "Immunolocalization of Lubricin in the Rat Periodontal Ligament during Experimental Tooth Movement," Acta Histochemica, Vol. 114, No. 7, 2012, pp. 700-704. doi:10.1016/j.acthis.2011.12.005

[5] R. Leonardi, N. F. Talic and C. Loreto, "MMP-13 (Collagenase 3) Immunolocalisation during Initial Orthodontic Tooth Movement in Rats," Acta Histochemica, Vol. 109, No. 3, 2007, pp. 215-220.

doi:10.1016/j.acthis.2007.01.002

[6] G. Cantarella, R. Cantarella, M. Caltabiano, N. Risuglia, R. Bernardini and R. Leonardi, "Levels of Matrix Metalloproteinases 1 and 2 in Human Gingival Crevicular Fluid during Initial Tooth Movement,” Americam Journal Orthodontics and Dentofacial Orthopedics, Vol. 130, No. 5, 2006, pp. 11-16. doi:10.1016/j.ajodo.2006.04.020

[7] V. Krishnan and Z. Davidovitch, "Cellular, Molecular, and Tissue-Level Reactions to Orthodontic Force,” Americam Journal Orthodontics and Dentofacial Orthopedics, Vol. 129, No. 4, 2006, pp. 1-32. doi:10.1016/j.ajodo.2005.10.007

[8] W. J. Rody, G. J. King and G. Gu, "Osteoclast Recruitment to Sites of Compression in Orthodontic Tooth Movement," Americam Journal Orthodontics and Dentofacial Orthopedics, Vol. 120, No. 5, 2001, pp. 477-489. doi:10.1067/mod.2001.118623

[9] H. Lucas, P. M. Bartold, A. A. Dharmapatni, C. A. Holding and D. R. Haynes, "Inhibition of Apoptosis in Periodontitis,” Journal of Dental Research, Vol. 89, No. 1, 2010, pp. 29-33. doi:10.1177/0022034509350708

[10] R. S. Masella and M. Meister, "Current Concepts in the Biology of Orthodontic Tooth Movement," Americam Journal Orthodontics and Dentofacial Orthopedics, Vol. 129, No. 4, 2006, pp. 458-468. doi:10.1016/j.ajodo.2005.12.013

[11] P. J. Brooks, D. Nilforoushan, M. F. Manolson, C. A. Simmons and S. G. Gong, "Molecular Markers of Early Orthodontic Tooth Movement," Angle Orthodontis, Vol. 79, No. 6, 2009, pp. 1108-1113. doi:10.2319/121508-638R.1

[12] B. F. Boyce and L. Xing, "Functions of RANKL/ RANK/ OPG in Bone Modeling and Remodeling," Archives Biochememical and Biophysics, Vol. 473, No. 2, 2008, pp. 139-146. doi:10.1016/j.abb.2008.03.018

[13] B. Chen, S. Ma, S. Yang, X. Xing, R. Gu and Y. Hu, "DR5 and DcR2 Are Expressed in Human Lumbar Intervertebral Discs,” Spine, Vol. 34, No. 19 , pp. 677-681. doi:10.1097/BRS.0b013e3181b4d4ee

[14] C. Loreto, G. Musumeci, A. Castorina, C. Loreto and G. Martinez, "Degenerative Disc Disease of Herniated Intervertebral Discs is Associated with Extracellular Matrix Remodeling, Vimentin-Positive Cells and Cell Death,” Annual of Anatomy, Vol. 193, No. 2, 2011, pp. 156-162. doi:10.1016/j.aanat.2010.12.001

[15] S. Colucci, G. Brunetti, F. P. Cantatore, A. Oranger, G. Mori, P. Pignataro, et al., "The Death Receptor DR5 is Involved in TRAIL-Mediated Human Osteoclast Apoptosis,” Apoptosis, Vol. 12, No. 9, 2007, pp. 1623-1632. doi:10.1007/s10495-007-0095-3

[16] H. Kumamoto and K. Ooya, "Expression of Tumor Necrosis Factor Alpha, TNF-Related Apoptosis-Inducing ligand, and Their Associated Molecules in Ameloblastomas," Journal of Oral Pathology and Medicine, Vol. 34, No. 5, 2005, pp. 287-294.

doi:10.1111/j.1600-0714.2005.00311.x

[17] C. Loreto, G. Barbagli, R. Djinovic, G. Vespasiani, M. L. Carnazza, R. Miano, et al., "Tumor Necrosis Factor-Related Apoptosis-Inducing Ligand (TRAIL) and Its Death Receptor (DR5) in Peyronie's Disease. A Biomolecular Study of Apoptosis Activation," Journal of Sexual Medicine, Vol. 2011, No. 1, 2011, pp. 109-115. doi:10.1111/j.1743-6109.2010.02003.x

[18] R. Leonardi, L. E. Almeida, P. C. Trevilatto and C. Loreto, "Occurrence and Regional Distribution of TRAIL and DR5 on Temporomandibular Joint Discs: Comparison of Disc Derangement with and without Reduction," Oral Surgery, Oral Medicine, Oral Pathology, Oral Radiology and Endodontics, Vol. 109, No. 2, 2009, pp. $244-$ 251. doi:10.1016/j.tripleo.2009.09.028

[19] Y. Ren, J. C. Maltha and A. M. Kuijpers-Jagtman, “The Rat as a Model for Orthodontic Tooth Movement-A Critical Review and a Proposed Solution,” European Journal Orthodontics, Vol. 26, No. 5, 2004, pp. 483-490. doi:10.1093/ejo/26.5.483

[20] R. Leonardi, M. C. Rusu, F. Loreto, C. Loreto and G. Musumeci, "Immunolocalization and Expression of Lubricin in the Bilaminar Zone of the Human Temporomandibular Disc,” Acta Histochemica, Vol. 114, No. 1, 2012, pp. 1-5. doi:10.1016/j.acthis.2010.11.011

[21] X. Li, L. Zhang, N. Wang, X. Feng and L. Bi, "Periodontal Ligament Remodeling and Alveolar Bone Resorption during Orthodontic Tooth Movement in Rats with Diabetes," Diabetes Technology and Therapy, Vol. 12, No. 1, 2010, pp. 65-73. doi:10.1089/dia.2009.0085

[22] R. Xie, A. M. Kuijpers-Jagtman and J. C. Maltha, “Osteoclast Differentiation and Recruitment during Early Stages of Experimental Tooth Movement in Rats," European Journal of Oral Sciences, Vol. 117, No. 1, 2009, pp. 43-50. doi:10.1111/j.1600-0722.2008.00588.X

[23] C. Falschlehner, C. H. Emmerich, B. Gerlach and H. Walczak, "TRAIL Signalling: Decisions between Life and Death,” International Journal of Biochemistry and Cell Biology, Vol. 39, No. 7-8, 2007, pp. 1462-1475.

[24] T. M. Baetu and J. Hiscott, "On the TRAIL to Apoptosis," Cytokine and Growth Factor Reviews, Vol. 13, No. 3, 2002, pp. 199-207. doi:10.1016/S1359-6101(02)00006-0

[25] B. R. Chaudhari, R. F. Murphy and D. K. Agrawal, "Following the TRAIL to Apoptosis," Immunology Research, Vol. 35, No. 3, 2006, pp. 249-262. doi:10.1385/IR:35:3:249

[26] C. Loreto, L. E. Almeida, M. R. Migliore, M. Caltabiano and R. Leonardi, "TRAIL, DR5 and Caspase 3-Dependent Apoptosis in Vessels of Diseased Human Temporomandibular Joint Disc. An Immunohistochemical Study," European Journal of Histochemistry, Vol. 54, No. 3, 2010, pp. 175-179. doi:10.4081/ejh.2010.e40 
[27] C. Loreto, L. E. Almeida, P. Trevilatto and R. Leonardi, “Apoptosis in Displaced Temporomandibular Joint Disc with and without Reduction: An Immunohistochemical Study," Journal of Oral Pathology and Medicine, Vol. 40, No. 1, 2011, pp. 103-110. doi:10.1111/j.1600-0714.2010.00920.x

[28] C. Loreto, G. Musumeci and R. Leonardi, "Chondrocyte-Like Apoptosis in Temporomandibular Joint Disc Internal Derangement as a Repair-Limiting Mechanism. An in Vivo Study," Histology and Histopathology, Vol. 24, No. 3, 2009, pp. 293-298. doi:10.1007/s00167-010-1215-0

[29] G. Musumeci, C. Loreto, M. L. Carnazza and G. Martinez, "Characterization of Apoptosis in Articular Cartilage Derived from the Knee Joints of Patients with Osteoarthritis,” Knee Surgery Sports Traumatology Arthroscopy, Vol. 19, No. 2, 2011, pp. 307-313. doi:10.1007/s00167-010-1215-0

[30] R. Caltabiano, R. Leonardi, G. Musumeci, G. Bartoloni, M. C. Rusu, L. E. Almeida and C. Loreto, "Apoptosis in Temporomandibular Joint Disc with Internal Derangement Involves Mitochondrial-Dependent Pathways. An in Vivo Study,” Acta Odontologica Scandinavica, Vol. 71, No. 3-4, pp. 577-583. doi:10.3109/00016357.2012.700060

[31] A. Bhardwaj and B. B. Aggarwal, "Receptor-Mediated Choreography of Life and Death,” Journal of Clinical Immunology, Vol. 23, No. 5, 2003, pp. 317-332. doi:10.1023/A:1025319031417
[32] L. J. Robinson, C. W. Borysenko and H. C. Blair, “Tumor Necrosis Factor Family Receptors Regulating Bone Turnover: New Observations in Osteoblastic and Osteoclastic Cell Lines," Annals of the New York Academy of Sciences, Vol. 1116, No. 1, 2007, pp. 432-443. doi:10.1196/annals.1402.025

[33] A. R. Sekhavat, K. Mousavizadeh, H. R. Pakshir and F. S. Aslani, "Effect of Misoprostol, a Prostaglandin E1 Analog, on Orthodontic Tooth Movement in Rats," American Journal of Orthodontics and Dentofacial Orthopedics, Vol. 122, No. 5, pp. 542-547. doi:10.1067/mod.2002.126153

[34] K. Yamasaki, Y. Shibata, S. Imai, Y. Tani, Y. Shibasaki and T. Fukuhara, "Clinical Application of Prostaglandin E1 (PGE1) upon Orthodontic Tooth Movement," American Journal of Orthodontics, Vol. 85, No. 6, 1984, pp. 508-518. doi:10.1016/0002-9416(84)90091-5

[35] B. J. Leiker, R. S. Nanda, G. F. Currier, R. I. Howe and P. K. Sinha, "The Effects of Exogenous Prostaglandins on Orthodontic Tooth Movement in Rats,” American Journal of Orthodontics and Dentofacial Orthopedics, Vol. 108, No. 4, 1995, pp. 380-388. doi:10.1016/S0889-5406(95)70035-8

[36] V. Cardile, G. Musumeci, E. Sicurezza, S. Caggia, M. C. Rusu, R. Leonardi and C. Loreto, "TRAIL and Its Receptors DR5 and DcR2 Expression, in Orthodontic Tooth Movement," Histology and Histopathology, Vol. 28, No. 7, 2013, pp. 933-940. 